\title{
The $\gamma$-open Open Topology for Function Spaces
}

\author{
S. Ganguly and Ritu Sen
}

\begin{abstract}
In this paper we have introduced the notion of $\gamma$ - open open topology and proved some properties which the topology does possess. We have also introduced the concept of convergence of nets in $\gamma H(X)$ (where $\gamma H(X)$ is the set of all self $\gamma$ - homeomorphisms on a topological space $X)$ and showed when $\gamma H(X)$ is complete.
\end{abstract}

\section{INTRODUCTION}

A set-set topology is one which is defined as follows : Let $(X, \tau)$ and $\left(Y, \tau^{*}\right)$ be two topological spaces. Let $\mathcal{U}$ and $\mathcal{V}$ be collections of subsets of $X$ and $Y$ respectively. Let $F \subset Y^{X}$ be a collection of functions from $X$ into $Y$. We define, for $U \in \mathcal{U}$ and $V \in \mathcal{V},(U, V)=\{f \in F: f(U) \subset V\}$. Let $S(U, V)=\{(U, V): U \in \mathcal{U}, V \in \mathcal{V}\}$. If $S(U, V)$ is a subbasis for a topology $\tau(U, V)$ on $F$, then $\tau(U, V)$ is called a set-set topology.

The most commonly discussed set-set topologies are the compact-open topology, $\tau_{c o}$, which was introduced in 1945 by R.Fox [4] and the point-open topology, $\tau_{p}$. For $\tau_{c o}, \mathcal{U}$ is the collection of all compact subsets of $X$ and $\mathcal{V}$ the collection of all open subsets of $Y$, while for $\tau_{p}, \mathcal{U}$ is the collection of all singletons in $X$ and $\mathcal{V}$ the collection of all open subsets of $Y$.

In section 2 of this paper, we shall introduce and discuss the $\gamma$ open-open topology for function spaces. We shall also show which of the desirable properties $\tau_{\gamma o o}$ possesses. In section 3 , we shall introduce the notion of convergence of nets in $\left(\gamma H(X), \tau_{\gamma o o}\right)$ (where $\gamma H(X)$ is the collection of all self $\gamma$-homeomorphisms on $X)$ and the completeness of $\gamma H(X)$.

Throughout this paper, $(X, \tau)$ (simply $X)$ and $\left(Y, \tau^{*}\right)$ always mean topological spaces. Let $S$ be a subset of $X$. The closure (resp. interior) of $S$ will be denoted by $\operatorname{cl}(S)($ resp. $\operatorname{int}(S))$.

A subset $S$ of $X$ is called a semi-open set [7] if $S \subseteq \operatorname{cl}(\operatorname{int}(S))$. The complement of a semi-open set is called a semi-closed set. The family of all semi-open sets in a topological space $(X, \tau)$ will be denoted by $S O(X)$. A subset $M(x)$ of a space $X$ is called a semi-neighborhood of a point $x \in X$

2000 Mathematics Subject Classification. Primary: 54C35.

Key words and phrases. $\gamma$-open sets, $\gamma$-open open topology, $\gamma H(X)$-the set of all self $\gamma$-homeomorphisms on $X, \gamma$ - convergence, $\gamma$-regular. 
if there exists a semi-open set $S$ such that $x \in S \subseteq M(X)$. In [6] Latif introduced the notion of semi-convergence of filters. Let $S(x)=\{A \in$ $S O(X): x \in A\}$ and let $S_{x}=\{A \subseteq X$ : there exists $\mu \subseteq S(x)$ such that $\mu$ is finite and $\cap \mu \subseteq A\}$. Then $S_{x}$ is called the semi-neighborhood filter at $x$. For any filter $\Gamma$ on $X$ we say that $\Gamma$ semi-converges to $x$ if and only if $\Gamma$ is finer than the semi-neighborhood filter at $x$.

Definition $1.1([5])$. A subset $U$ of $X$ is called a $\gamma$ open set if whenever a filter $\Gamma$ semi-converges to $x$ and $x \in U, U \in \Gamma$. The complement of a $\gamma$ open set is called a $\gamma$ - closed set.

The intersection of all $\gamma$ - closed sets containing $A$ is called the $\gamma$-closure of $A$, denoted by $c l_{\gamma}(A)$.A subset $A$ is $\gamma$ - closed iff $A=c l_{\gamma}(A)$. We denote the family of all $\gamma$ - open sets of $(X, \tau)$ by $\tau^{\gamma}$. It is shown in [8] that $\tau^{\gamma}$ is a topology on $X$. In a topological space $(X, \tau)$, it is always true that $\tau \subseteq S(X) \subseteq \tau^{\gamma}$.

Example 1.2. We now give examples of $\gamma$ - open sets.

Let $X=\{0,1,2,3\}$,

$\tau=\{\phi,\{0\},\{1\},\{0,1\},\{0,2\},\{0,1,2\}, X\}$.

Now, $\{0,2\}$ and $\{0,3\}$ are semi-open sets and $\{0\}$ is an element of $S_{0}$. For any filter $\Gamma$ on $X$, if $\Gamma$ semi-converges to 0 , since $\Gamma$ includes $S_{0}$, then $\{0\}$ is a $\gamma$-open set.Also $\{3\}$ is a $\gamma$-open set which is not open in $X$.

Remark 1.3. Every open set of a topological space $X$ is a $\gamma$ - open set but the converse may not be true.

Definition 1.4 ([8]). A function $f: X \rightarrow Y$ is $\gamma$ - continuous if the inverse image of every open set of $Y$ is $\gamma$ - open in $X$.

The set of all $\gamma$-continuous functions from $X$ into $Y$ is denoted by $\gamma C(X, Y)$.

Definition $1.5([8])$. A function $f: X \rightarrow Y$ is said to be $\gamma$ - irresolute if the inverse image of every $\gamma$ - open set of $Y$ is $\gamma$ - open in $X$.

Definition 1.6. A function $f: X \rightarrow Y$ is said to be $\gamma$-homeomorphism if it is a bijection so that the image and the inverse image of $\gamma$-open sets are $\gamma$-open.

The collection of all $\gamma$ - homeomorphisms from $X$ into $Y$ is denoted by $\gamma H(X, Y)$.

Definition $1.7([5])$. A point $x \in X$ is said to be a $\gamma$-interior point of $A$ if there exists a $\gamma$ - open set $U$ containing $x$ such that $U \subseteq A$.

The set of all $\gamma$-interior points of $A$ is said to be $\gamma$ - interior of $A$ and is denoted by $\operatorname{int}_{\gamma}(A)$.

Theorem 1.8 ([5]). For a subset $A$ of a space $X, \operatorname{int}_{\gamma}(X \backslash A)=X \backslash c l_{\gamma}(A)$. 


\section{The $\gamma$-Open Open Topology}

Let $\mathcal{U}$ be the collection of all $\gamma$ - open sets in $X$ and $\mathcal{V}$ be the collection of all open sets in $Y$, then $S_{\gamma O O}=S(U, V)$ where $U \in \mathcal{U}$ and $V \in \mathcal{V}$ is the subbasis for a topology, $\tau_{\gamma o o}$, on any $F \subset Y^{X}$, which is called the $\gamma$ - open open topology.

We now examine some of the properties of function spaces the $\gamma$ - open open topology possesses.

Theorem 2.1. Let $F \subset Y^{X}$. If $\left(Y, \tau^{*}\right)$ is $T_{i}$, for $i=0,1,2$; then $\left(F, \tau_{\text {yoo }}\right)$ is $T_{i}$, for $i=0,1,2$.

Proof. We shall show the case $i=2$, the other cases are done similarly. Let $i=2$. Let $f, g \in F$ be such that $f \neq g$. Then there exists some $x \in X$ such that $f(x) \neq g(x)$. If $Y$ is $T_{2}$, then there exists disjoint open sets $U$ and $V$ in $Y$ such that $f(x) \in U$ and $g(x) \in V$. Both $f$ and $g$ are $\gamma$-continuous, so there are $\gamma$ - open sets $M$ and $N$ in $X$ with $x \in M \cap N, f(M) \subset U$ and $g(N) \subset V$. Hence, $f \in(M, U), g \in(N, V)$ and $(M, U) \cap(N, V)=\phi$. Thus

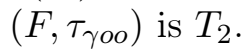

A topology $\tau^{*}$ on $F \subset Y^{X}$ is called an admissible [1] topology for $F$ provided the evaluation map $E:\left(F, \tau^{*}\right) \times(X, \tau) \rightarrow\left(Y, \tau^{\prime}\right)$ defined by $E(f, x)=f(x)$ is continuous.

Theorem 2.2. If $F \subset C(X, Y)$, then $\tau_{\text {yoo }}$ is admissible for $F$.

Proof. Let $F \subset C(X, Y)$. Let $V \in \tau^{\prime}$ and $(f, p) \in E^{-1}(V)$. Then $f(p) \in V$. Since $f$ is continuous, there exists some $U \in \tau$ such that $p \in U$ and $f(U) \subset$ $V$. So $(f, p) \in(U, V) \times U$. Since every open set is a $\gamma$ open set, $U$ is a $\gamma$ -open set as well as an open set. If $(g, y) \in(U, V) \times U$, then $g(U) \subset V$ and $y \in U$. So $g(y) \in V$. Hence $(U, V) \times U \subset E^{-1}(V)$. Therefore $\tau_{\gamma o o}$ is admissible for $F$.

Remark 2.3. The sets of the form $(U, V)$ where both $U$ and $V$ are $\gamma$-open sets in $X$ form a subbasis for $\left(\gamma H(X), \tau_{\gamma o o}\right)$.

Let $(G, \circ)$ be a group such that $(G, T)$ is a topological space, then $(G, T)$ is a topological group provided the two maps are continuous 1) $m: G \times G \rightarrow G$ is defined by $m\left(g_{1}, g_{2}\right)=g_{1} \circ g_{2}$ and 2) $\Phi: G \rightarrow G$ defined by $\Phi(g)=g^{-1}$. If only the first map is continuous, then we call $(G, T)$ a quasi-topological group [9].

Note that $\gamma H(X)$ with the binary operation $\circ$, compositions of functions, and identity element $e$, is a group.

Theorem 2.4. Let $X$ be a topological space and let $G$ be a subgroup of $\gamma H(X)$. Then $\left(G, \tau_{\gamma o o}\right)$ is a topological group.

Proof. Let $X$ be a topological space and $G$ be a subgroup of $\gamma H(X)$. We have to prove that the two maps $m: G \times G \rightarrow G$ defined by $m\left(g_{1}, g_{2}\right)=g_{1} \circ g_{2}$ and $\Phi: G \rightarrow G$ defined by $\Phi(g)=g^{-1}$ are continuous. 
Let $(U, V)$ be a subbasic open set in $\tau_{\gamma o o}$ such that both $U$ and $V$ are $\gamma$ open sets. Let $(f, g) \in m^{-1}((U, V))$. Then $f \circ g(U) \subset V$ and $g(U) \subset f^{-1}(V)$. So $(f, g) \in(g(U), V) \times(U, g(U)) \in \tau_{\gamma o o} \times \tau_{\gamma o o}$. But $(g(U), V) \times(U, g(U)) \subset$ $m^{-1}((U, V))$. Thus $m$ is continuous.

Now the inverse map $\Phi: G \rightarrow G$ is bijective and $\Phi^{-1}=\Phi$. Thus in order to show that $\Phi$ is continuous, it is sufficient to show that $\Phi$ is an open

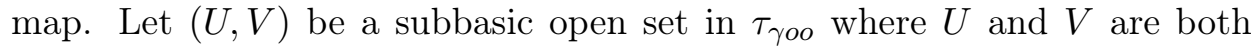
$\gamma$ - open sets. Now $\Phi((U, V))=((X \backslash V, X \backslash U))$; since we are dealing with $\gamma$ - homeomorphisms. Now, if $C$ and $D$ are $\gamma$-closed sets, then int ${ }_{\gamma} C$ and int $_{\gamma} D$ are $\gamma$ - open sets (using Theorem 1.8). Thus, since $(X \backslash V),(X \backslash U)$ are $\gamma$ - closed sets, $\operatorname{int}_{\gamma}(X \backslash V)$, int $_{\gamma}(X \backslash U)$ are $\gamma$ - open sets. Again since $G$ is a set of $\gamma$ - homeomorphisms, $(X \backslash V, X \backslash U)=\left(\operatorname{int}_{\gamma}(X \backslash V)\right.$ int $\left._{\gamma}(X \backslash U)\right)$ but this is in $\tau_{\gamma o o}$. Therefore $\Phi(U, V)$ is an open set in $\tau_{\gamma o o}$. So, $\Phi$ is open and our theorem is proved.

\section{Completeness of $\left(\gamma H(X), \tau_{\gamma o o}\right)$}

We now introduce the notion of convergence of nets in $\left(\gamma H(X), \tau_{\gamma o o}\right)$ and the completeness of $\left(\gamma H(X), \tau_{\gamma o o}\right)$. For this purpose, we require the following definitions and theorems.

Definition 3.1. A net in a set $X$ (where $X$ is a topological space) is a map $x: \Lambda \rightarrow X$ ( $\Lambda$ is a directed set). We often write such a net by the symbol $\left\{x_{\lambda}: \lambda \in \Lambda\right\}$ writing $x_{\lambda}$ instead of $x(\lambda)$.

Definition 3.2. A net $\left\{x_{\lambda}: \lambda \in \Lambda\right\}$ in $X$ is said to be converge to a limit $x \in X$ (in symbol $x_{\lambda} \rightarrow x$ ) if for every neighborhood $V$ of $x, \exists$ a $\lambda_{0} \in \Lambda$ such that $\lambda \geq \lambda_{0}$ implies $x_{\lambda} \in V$.

Definition 3.3. A net $\left\{x_{\lambda}: \lambda \in \Lambda\right\}$ in $X$ is said to $\gamma$ - converge to a limit $x \in X$ (in symbol $x_{\lambda} \rightarrow^{\gamma} x$ ) if for every $\gamma$ - open set $V$ containing $x, \exists \lambda_{0} \in \Lambda$ such that $\lambda \geq \lambda_{0}$ implies $x_{\lambda} \in V$. We often denote this by $\gamma \lim _{\lambda} x_{\lambda}=x$.

Theorem 3.4. A function $f: X \rightarrow Y$ (where $X$ and $Y$ are topological spaces) is $\gamma$-irresolute at a point $x \in X$ iff for any net $\left\{x_{\lambda}: \lambda \in \Lambda\right\}$ in $X$ $\gamma$-converging to $x$, the net $\left\{f\left(x_{\lambda}\right): \lambda \in \Lambda\right\} \gamma$-converges to $f(x)$ in $Y$.

Proof. First assume that $f$ is $\gamma$ - irresolute at $x \in X$. Let $\left\{x_{\lambda}: \lambda \in \Lambda\right\}$ be a net in $X \gamma$-converging to $x$. Let $V$ be a $\gamma$ - open set in $Y$ containing $f(x)$. Now $\exists$ a $\gamma$ - open set $U$ containing $x$ in $X$ such that $f(U) \subset V$ Now $\left\{x_{\lambda}: \lambda \in \Lambda\right\} \gamma$ - converges to $x$ implies $\exists \lambda_{0} \in \Lambda$ such that $x_{\lambda} \in U, \forall \lambda \geq \lambda_{0}$. Hence, $\forall \lambda \geq \lambda_{0}, f\left(x_{\lambda}\right) \in V$. This shows that $\left\{f\left(x_{\lambda}\right): \lambda \in \Lambda\right\}$ lies eventually in $V$ and hence it $\gamma$ - converges to $f(x)$.

To prove the converse, assume that $f$ is not $\gamma$ - irresolute at $x$. Then $\exists$ a $\gamma$ - open set $W$ containing $f(x)$ in $Y$ such that from every $\gamma$ - open set $U$ containing $x \in X, \exists$ an element $x_{U}$ with $f\left(x_{U}\right) \notin W$. Let $\gamma \mathcal{N}_{x}$ be the $\gamma$ neighborhood system at $x$. So, $\left\{x_{U}: U \in \gamma \mathcal{N}_{x}\right\}$ is a net in $X \gamma$ - converging 
to $x$, but the net $\left\{f\left(x_{U}\right): U \in \gamma \mathcal{N}_{x}\right\}$ in $Y$ does not lie eventually in $W$ and consequently it cannot $\gamma$ - converge to $f(x)$.

Theorem 3.5. Let $\left\{h_{\nu}: \nu \in \mathcal{V}\right\}$ be a net in the group $\gamma H(X)$ of self $\gamma$ - homeomorphisms of a topological space $X$. Then $h_{\nu} \rightarrow h$ in $\tau_{\gamma o o}$ iff $h_{\nu}\left(x_{\delta}\right) \rightarrow^{\gamma} h(x)$ whenever $x_{\delta} \rightarrow^{\gamma} x$ in $X$.

Proof. First assume that, $h_{\nu} \rightarrow h$ in $\tau_{\gamma o o}$. Let $(U, V)(U, V$ both are $\gamma$ - open sets of $X)$ be an open set in $\left(\gamma H(X), \tau_{\gamma o o}\right)$ containing $h$. Then $\exists \nu_{0} \in \mathcal{V}$ such that $h_{\nu} \in(U, V), \forall \nu \geq \nu_{0}$ ie, $h_{\nu}(U) \subset V, \forall \nu \geq \nu_{0}$. Now, let $x_{\delta} \rightarrow^{\gamma} x$ in $X$. Then for every $\gamma$ - open set $U$ containing $x, \exists \delta_{0} \in D$ such that $x_{\delta} \in U$, $\forall \delta \geq \delta_{0}$. Hence, $\forall \nu \geq \nu_{0}, \delta \geq \delta_{0} ; h_{\nu}\left(x_{\delta}\right) \in V$. Also, $h(x) \in V$. Hence $\left\{h_{\nu}\left(x_{\delta}\right): \nu \in \mathcal{V}, \delta \in D\right\} \gamma$ - converges to $h(x)$ whenever $x_{\delta} \rightarrow^{\gamma} x$.

Next, if possible, let $h_{\nu} \nrightarrow h$ in $\tau_{\gamma o o}$. Then $\exists$ a neighborhood $(U, V)(U$, $V$ both are $\gamma$ - open sets of $X)$ containing $h$ such that $\forall \nu \in \mathcal{V}, h_{\nu} \notin(U, V)$ ie, $h_{\nu}(U) \not \subset V$. So from every $\gamma$ - open set $U$ containing $x, \exists$ an element $x_{U}$ with $h_{\nu}\left(x_{U}\right) \notin V$. Let $\gamma \mathcal{N}_{x}$ be the $\gamma$ - neighborhood system at $x$. Now $h \in(U, V)$ implies $h(U) \subset V$. Hence, $\forall \nu \in \mathcal{V}, U \in \gamma \mathcal{N}_{x}, h_{\nu}\left(x_{U}\right) \notin h(U)$, ie, $h_{\nu}\left(x_{U}\right) \nrightarrow^{\gamma} h(x)$. Contrapositively, we can say that whenever $h_{\nu}\left(x_{U}\right) \rightarrow^{\gamma}$ $h(x)$ for $x_{U} \rightarrow^{\gamma} x ; h_{\nu} \rightarrow h$ in $\tau_{\gamma o o}$

Now we define a uniformity $\mathcal{U}_{o}$ on $\gamma H(X)$ by defining $(x, y) \in U_{o}$ if $x y^{-1} \in U$ and $y x^{-1} \in U$ where $U$ is a neighborhood of the identity in $\gamma H(X)$. Then $\left(\gamma H(X), \mathcal{U}_{o}\right)$ becomes a uniform space.

Definition 3.6. A net $\left\{h_{\nu}: \nu \in \mathcal{V}\right\}$ in $\left(\gamma H(X), \mathcal{U}_{o}\right)$ is called a Cauchy net if for each $U \in \mathcal{U}_{o}, \exists$ a $\nu_{0} \in \mathcal{V}$ such that $\nu_{1}, \nu_{2}>\nu_{0}$ implies $\left(h_{\nu_{1}}, h_{\nu_{2}}\right) \in U$. If every Cauchy net in $\gamma H(X)$ converges (has a limit in $\gamma H(X)$ ), then $\gamma H(X)$ will be called complete (in the structure $\mathcal{U}_{o}$ ).

Definition 3.7. A topological space $X$ is said to be $\gamma$-regular if for each open set $U$ of $X$ and each $x \in U$, there exists a $\gamma$ - open set $V$ in $X$, such that $x \in V \subseteq U$.

Example 3.8. We give an example of a $\gamma$ - regular space which is not regular.Consider

$X=\{0,1,2,3,4,5\}$

$\tau=\{\phi,\{0,2,4\},\{3,5\},\{0,2,3,4,5\},\{2,4\},\{2,3,4,5\}, X\}$

Now $\{1\}$ is a closed set and $0 \notin\{1\}$. But 0 and $\{1\}$ cannot be strongly separated. Hence, $X$ is not regular. Now, $\{3,5\},\{0,2,4\},\{1,2,4\},\{1,3,5\}$, $\{0,2,3,4,5\}$ are semi-open sets. Also, $\{3,5\} \in S_{3},\{3,5\} \in S_{5},\{2,4\} \in S_{2}$, $\{2,4\} \in S_{4},\{0,2,4\} \in S_{0},\{1\} \in S_{1}$. For any open neighborhood $U_{0}$ of 0 , $0 \in\{0,2,4\} \subseteq U_{0}$; for any open neighborhood $U_{1}$ of $1,1 \in\{1\} \subset U_{1}$; for any open neighborhood $U_{2}$ of $2,2 \in\{2,4\} \subseteq U_{2}$; for any open neighborhood $U_{3}$ of $3,3 \in\{3,5\} \subseteq U_{3}$; for any open neighborhood $U_{4}$ of $4,4 \in\{2,4\} \subseteq U_{4}$; for any open neighborhood $U_{5}$ of $5,5 \in\{3,5\} \subseteq U_{5}$. Hence $X$ is $\gamma$ - regular.

Theorem 3.9. $\left(\gamma H(X), \tau_{\gamma o o}\right)$ is complete if $X$ is $\gamma$-regular and complete. 
Proof. Let $\left\{h_{\nu}: \nu \in \mathcal{V}\right\}$ be a Cauchy net in $\gamma H(X)$ (relative to $\mathcal{U}_{o}$ ) ie, $h_{\nu} h_{\mu}{ }^{-1} \rightarrow$ identity and $h_{\mu} h_{\nu}{ }^{-1} \rightarrow$ identity, for $\mu, \nu \in \mathcal{V}$.Also, for each $x \in X,\left\{h_{\nu}(x): \nu \in \mathcal{V}\right\}$ is a Cauchy net in $X$ and hence converges for each $x \in X$. Let its limit be $h(x)$. We will show that whenever a net $\left\{x_{\delta}: \delta \in D\right\}$ in $X \gamma$ - converges to $x \in X$, the net $h_{\nu}\left(x_{\delta}\right) \rightarrow^{\gamma} h(x)$.If possible, let $h_{\nu}\left(x_{\delta}\right) \nrightarrow^{\gamma} h(x)$ and suppose that $h_{\nu}\left(x_{\delta}\right) \rightarrow^{\gamma} y \neq h(x)$. Since $X$ is $\gamma$ - regular, $h_{\nu}\left(x_{\delta}\right) \rightarrow y \neq h(x)$. Now, $\lim _{\nu, \delta} h_{\nu}\left(x_{\delta}\right)=y, \lim _{\delta} h_{\nu}\left(x_{\delta}\right)=$ $h_{\nu}(x) \rightarrow h(x)$. Since, $\lim _{\nu, \delta} h_{\nu}\left(x_{\delta}\right)=\lim _{\nu} \lim _{\delta} h_{\nu}\left(x_{\delta}\right)$, therefore $y=h(x)$ and this contradiction proves that $h_{\nu}\left(x_{\delta}\right) \rightarrow^{\gamma} h(x)$. Thus $h_{\nu} \rightarrow h$ in $\tau_{\gamma o o}$. We now show that $h$ is $\gamma$ - irresolute at the point $x \in X$. We know that if $x_{\delta} \rightarrow^{\gamma} x$, then $h_{\nu}\left(x_{\delta}\right) \rightarrow^{\gamma} h(x)$. Therefore, $\gamma \lim _{\nu, \delta} h_{\nu}\left(x_{\delta}\right)=\gamma \lim _{\nu} \gamma \lim _{\delta} h_{\nu}\left(x_{\delta}\right)=$ $\gamma \lim _{\delta} h\left(x_{\delta}\right)$ and hence $h\left(x_{\delta}\right) \rightarrow^{\gamma} h(x)$. Thus we have, whenever $x_{\delta} \rightarrow^{\gamma} x$, $h\left(x_{\delta}\right) \rightarrow^{\gamma} h(x)$. Using Theorem 3.4 we can show that $h$ is $\gamma$ - irresolute at $x \in X$. Since the conditions on $h$ are equivalent to the same conditions on $h^{-1}$, we have $h \in \gamma H(X)$ and hence $\left(\gamma H(X), \tau_{\gamma o o}\right)$ is complete.

\section{REFERENCES}

[1] R. Arens, Topologies for Homeomorphism Groups, Amer. J. Math 68(1946), 593-610.

[2] P. Fletcher and W. Lindgren, Quasi Uniform Spaces, Lecture Notes in Pure and Applied Mathematics 77, Marcel Dekker, 1982.

[3] P. Fletcher, Homeomorphism Groups with the Topology of Quasi-Uniform Convergence, Arch. Math. 22(1971), 88-92.

[4] R. Fox, On Topologies for Function Spaces, Bull. Amer. Math. Soc. 51(1945), 429432.

[5] R.M. Latif, Characterizations and Applications of $\gamma$-open sets, communicated.

[6] R.M. Latif, Semi convergence of Filters and Nets, Math. J. of Okayama University, Vol. 4(1999), 103-109.

[7] N. Levine, Semi open sets and semi continuity in Topological spaces, Amer. Math. Monthly, 70(1963), 36-41.

[8] W.K. Min, $\gamma$ - sets and $\gamma$ - continuous functions, Int. J. Math. Math. Sci., Vol. 31, No. 3(2002), 177-181.

[9] M. Murdeswar and S. Naimpally, Quasi Uniform Topological Spaces, Noordoff, 1966.

[10] S. Naimpally, Fuction Spaces of Quasi Uniform Spaces, Indag. Math. 68(1965), 768771.

[11] K. Porter, The Open Open Topology for Function Spaces, Inter. J. Math and Math. Sci. 18(1993), 111-116.

[12] K. Porter, The Regular Open-Open Topology for Function Spaces, Inter. J. Math and Math. Sci. 19(1996), 299-302. 
S. Ganguly

Department of Pure Mathematics

University of CAlCUTta

35, Ballygunge Circular Road

KOLKATA-700019

INDIA

E-mail address: gangulys04@yahoo.co.in

\section{Ritu Sen}

70E, Ananda Palit Road

KOLKATA-700014

INDIA

E-mail address: ritu_sen29@yahoo.co.in 